\title{
WASP-103 b: a new planet at the edge of tidal disruption ${ }^{\star}$
}

\author{
M. Gillon ${ }^{1}$, D. R. Anderson ${ }^{2}$, A. Collier-Cameron ${ }^{3}$, L. Delrez ${ }^{1}$, C. Hellier ${ }^{2}$, E. Jehin ${ }^{1}$, M. Lendl ${ }^{4}$, P. F. L. Maxted ${ }^{2}$ \\ F. Pepe ${ }^{4}$, D. Pollacco ${ }^{5}$, D. Queloz ${ }^{6,4}$, D. Ségransan ${ }^{4}$, A. M. S. Smith ${ }^{7,2}$, B. Smalley ${ }^{2}$, J. Southworth ${ }^{2}$, \\ A. H. M. J. Triaud ${ }^{8,4}$, S. Udry ${ }^{4}$, V. Van Grootel ${ }^{1}$, and R. G. West ${ }^{5}$ \\ ${ }^{1}$ Institut d'Astrophysique et de Géophysique, Université de Liège, Allée du 6 août 17, Sart Tilman, 4000 Liège 1, Belgium \\ e-mail: michael.gillon@ulg.ac.be \\ 2 Astrophysics Group, Keele University, Staffordshire, ST5 5BG, UK \\ 3 SUPA, School of Physics and Astronomy, University of St. Andrews, North Haugh, Fife, KY16 9SS, UK \\ 4 Observatoire de Genève, Université de Genève, 51 Chemin des Maillettes, 1290 Sauverny, Switzerland \\ 5 Department of Physics, University of Warwick, Coventry CV4 7AL, UK \\ ${ }^{6}$ Cavendish Laboratory, Department of Physics, University of Cambridge, JJ Thomson Avenue, Cambridge, CB3 0HE, UK \\ 7 N. Copernicus Astronomical Centre, Polish Academy of Sciences, Bartycka 18, 00-716 Warsaw, Poland \\ 8 Department of Physics and Kavli Institute for Astrophysics \& Space Research, Massachusetts Institute of Technology, Cambridge, \\ MA 02139, USA
}

Received 8 November 2013 / Accepted 9 January 2014

\section{ABSTRACT}

\begin{abstract}
We report the discovery of WASP-103 b, a new ultra-short-period planet $(P=22.2 \mathrm{~h})$ transiting a $12.1 \mathrm{~V}$-magnitude F8-type mainsequence $\operatorname{star}\left(1.22 \pm 0.04 M_{\odot}, 1.44_{-0.03}^{+0.05} R_{\odot}, T_{\text {eff }}=6110 \pm 160 \mathrm{~K}\right)$. WASP-103 b is significantly more massive $\left(1.49 \pm 0.09 M_{\mathrm{Jup}}\right)$ and larger $\left(1.53_{-0.07}^{+0.05} R_{\mathrm{Jup}}\right)$ than Jupiter. Its large size and extreme irradiation $\left(\sim 9 \times 10^{9} \mathrm{erg} \mathrm{s}^{-1} \mathrm{~cm}^{-2}\right)$ make it an exquisite target for a thorough atmospheric characterization with existing facilities. Furthermore, its orbital distance is less than $20 \%$ larger than its Roche radius, meaning that it might be significantly distorted by tides and might experience mass loss through Roche-lobe overflow. It thus represents a new key object for understanding the last stage of the tidal evolution of hot Jupiters.
\end{abstract}

Key words. planetary systems - stars: individual: WASP-103 - techniques: photometric - techniques: radial velocities techniques: spectroscopic

\section{Introduction}

Ever since the historical detection of the transits of HD $209458 \mathrm{~b}$ (Charbonneau et al. 2000; Henry et al. 2000), the ultra-shortperiod (a few days, or even less) transiting giant planets have played a pivotal role for exoplanetary science. These transiting hot Jupiters undergo irradiation orders of magnitude larger than any solar system planets (Fortney et al. 2007), and are also subject to intense gravitational and magnetic fields (Correia \& Laskar 2011; Chang et al. 2010). Their eclipsing configuration provides us with a unique opportunity to study their response to such extreme conditions and to improve substantially our knowledge of planetary structure, chemical composition and physical mechanisms. Notably, it allows us to study the last stages of their tidal evolution, provided giant planets close to tidal disruption are found to be transiting bright stars.

Our WASP transit survey (Pollacco et al. 2006) actively searches for ultra-short-period giant planets. Despite a maximized detection probability (higher transit probability, more frequent transits), planets with a period shorter than $1.2 \mathrm{~d}$ represent only a few percent of the total WASP harvest, demonstrating their rarity (Hellier et al. 2012). This small subgroup has so far been composed of WASP-12 b (Hebb et al. 2009), WASP-18 b (Hellier et al. 2009), WASP-19b (Hebb et al. 2010), and WASP-43 b (Hellier et al. 2011a). Among these four planets,

* The photometric and radial velocity time-series are only available at the CDS via anonymous ftp to cdsarc.u-strasbg. fr $(130.79 .128 .5)$ or via

http://cdsarc.u-strasbg.fr/viz-bin/qcat?J/A+A/562/L3
WASP-12 b and WASP-19b are the only two gas giants close to tidal disruption known to transit a bright star. We report here our discovery of a third planet of this ultra-rare kind, WASP-103 b, which orbits a $12.1 \mathrm{~V}$-magnitude F-type star at only $\sim 3$ stellar radii.

Section 2 presents our discovery and follow-up data. In Sect. 3, we briefly present the spectroscopic determination of the stellar properties and the derivation of the system parameters through a combined analysis of all our data. We discuss our discovery in Sect. 4.

\section{Observations}

\subsection{WASP transit detection photometry}

The host star WASP-103 (1SWASPJ163715.59+071100.0 = 2MASS16371556+0711000; $V=12.1, K=10.8)$ was observed by the southern station of the WASP survey (Hellier et al. 2011b) during the 2010, 2011, and 2012 observing seasons, covering the intervals 2010 May 15 to Aug. 16, 2011 Mar. 26 to Aug. 20, and 2012 Mar. 25 to Jun. 28. The 11565 pipeline-processed photometric measurements were detrended and searched for transits using the methods described by Collier-Cameron et al. (2006). The selection process (Collier-Cameron et al. 2007) elected WASP-103 as a high priority candidate presenting a periodic transit-like signature with a period of 0.926 days. Figure 2.1 (top panel) presents the WASP photometry folded on the best-fit transit ephemeris.

A search for periodic modulation was applied to the WASP photometry of WASP-103, using the method described in 


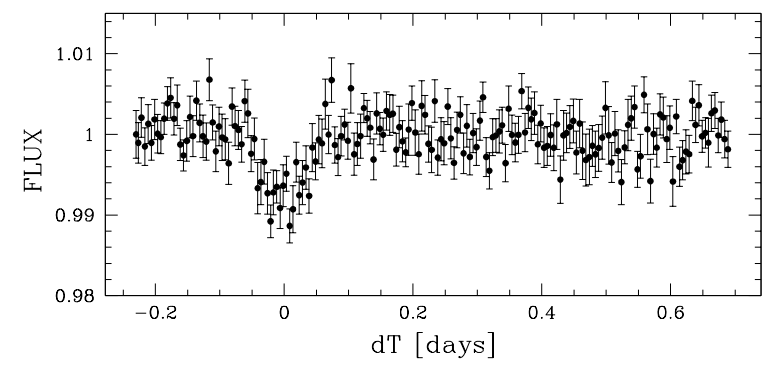

Fig. 1. WASP photometry for WASP-103 period-folded on the bestfit transit ephemeris from the transit search algorithm presented in Collier-Cameron et al. (2006), and binned per $0.005 \mathrm{~d}$ intervals.

Table 1. Summary of follow-up transit photometry obtained for WASP-103.

\begin{tabular}{cccccc}
\hline \hline Night & Telescope & Filter & $N$ & $\begin{array}{c}T_{\exp } \\
(\mathrm{s})\end{array}$ & Baseline function \\
\hline 2013 Jun. 15-16 TRAPPIST & $B B$ & 802 & 7 & $p\left(t^{1}+f^{1}\right)+o$ \\
2013 Jun. 28-29 & Euler & Gunn- $r^{\prime}$ & 103 & 120 & $p\left(t^{1}+f^{1}+b^{2}+x y^{1}\right)$ \\
2013 Jul. 11-12 & Euler & Gunn- $r^{\prime}$ & 105 & 80 & $p\left(t^{1}+b^{1}\right)$ \\
2013 Jul. 23-24 & TRAPPIST & $B B$ & 941 & 9 & $p\left(t^{2}\right)+o$ \\
2013 Aug. 4-5 & TRAPPIST & $B B$ & 935 & 10 & $p\left(t^{2}\right)+o$ \\
\hline
\end{tabular}

Notes. $N=$ number of measurements. $T_{\exp }=$ exposure time. $\mathrm{BB}=$ blue-blocking filter. The baseline functions are the analytical functions used to model the photometric baseline of each light curve (see G13). $o$ denotes an offset fixed at the time of the meridian flip, and $p\left(\alpha^{\beta}\right)$ a $\beta$-order polynomial function of time $(\alpha=t)$, full width at half maximum $(\alpha=f)$, background $(\alpha=b)$, or stellar position on the detector $(\alpha=x y)$.

Maxted et al. (2011). No periodic signal was found down to the 3 mmag amplitude.

\subsection{Follow-up photometry}

In 2013, we observed three transits of WASP-103 b with the robotic $60 \mathrm{~cm}$ telescope TRAPPIST (TRAnsiting Planets and PlanetesImals Small Telescope; Jehin et al. 2011) located at the ESO La Silla Observatory in the Atacama Desert, Chile. These transits were observed in a blue-blocking filter ${ }^{1}$ that has a transmittance $>90 \%$ from $500 \mathrm{~nm}$ to beyond $1000 \mathrm{~nm}$. Two transits were also observed in the Gunn- $r^{\prime}$ filter with the EulerCam CCD camera at the 1.2-m Euler Telescope at La Silla Observatory (Lendl et al. 2012).

For these follow-up eclipse monitorings, the procedures for the observation and data reduction were similar to those described by Gillon et al. (2013, hereafter G13) for WASP-64 and WASP-72, and we refer to this paper for more details. Table 1 presents a summary of our follow-up photometric time-series obtained for WASP-103. The resulting light curves are shown in Fig. 2.2.

\subsection{Spectroscopy and radial velocities}

We gathered 18 spectroscopic measurements with the CORALIE spectrograph mounted on Euler to confirm the planetary nature of the body eclipsing WASP-103 and to measure its mass. These spectra were obtained in the time interval between 2013 Jun. 1 to 2013 Sep. 10, each with an exposure time of $30 \mathrm{~min}$. Radial velocities (RVs) were computed from the spectra by

\footnotetext{
1 http://www . astrodon.com/products/filters/exoplanet/
}
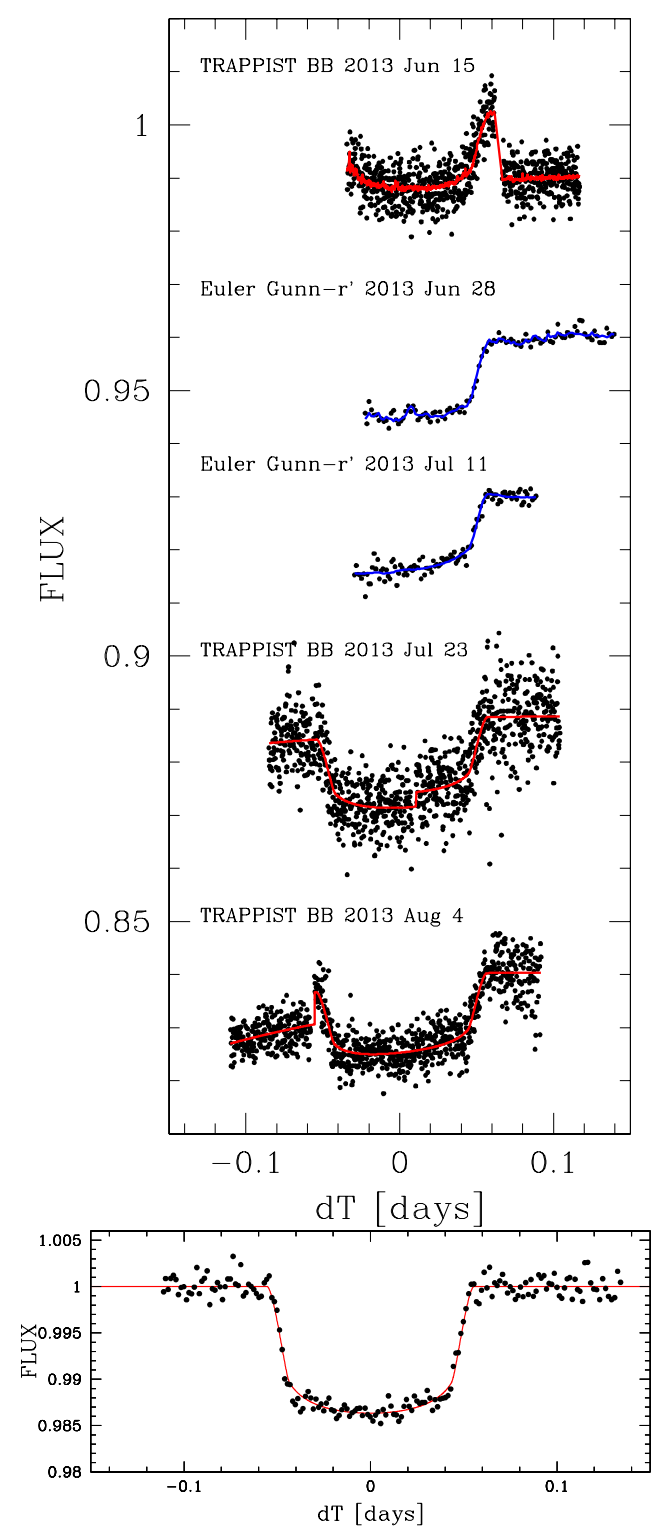

Fig. 2. Top: follow-up transit photometry for WASP-103 b. For each light curve, the best-fit transit+baseline model deduced in our global analysis (Sect. 3.2) is superimposed. The light curves are shifted along the $y$-axis for clarity. $\mathrm{BB}=$ blue-blocking filter. The flux offset visible in each BB light curve corresponds to the meridian flip of the TRAPPIST German equatorial mount, resulting in a different position of the stars on the chip. Bottom: same follow-up photometry divided by the bestfit baseline functions, period-folded on the best-fit transit ephemeris, and binned in 2 min intervals. Each point corresponds to the mean flux value for the corresponding time interval. The best-fit transit model is overplotted in red.

weighted cross-correlation (Baranne et al. 1996) with a numerical G2-spectral template that provides close to optimal precisions for late-F to early-K dwarfs, from our experience.

The RV time-series shows a sinusoidal signal with a period and phase in excellent agreement with those deduced from the WASP transit detection, and with an amplitude consistent with a planetary-mass object orbiting a main-sequence star (Fig. 3). To confirm that the RV signal originates from a planet-mass object orbiting the star, we analyzed the CORALIE cross-correlation functions (CCF) using the line-bisector technique described in Queloz et al. (2001). The bisector spans were revealed to be stable, their standard deviation being close to their average error 
M. Gillon et al.: WASP-103 b: a new planet at the edge of tidal disruption
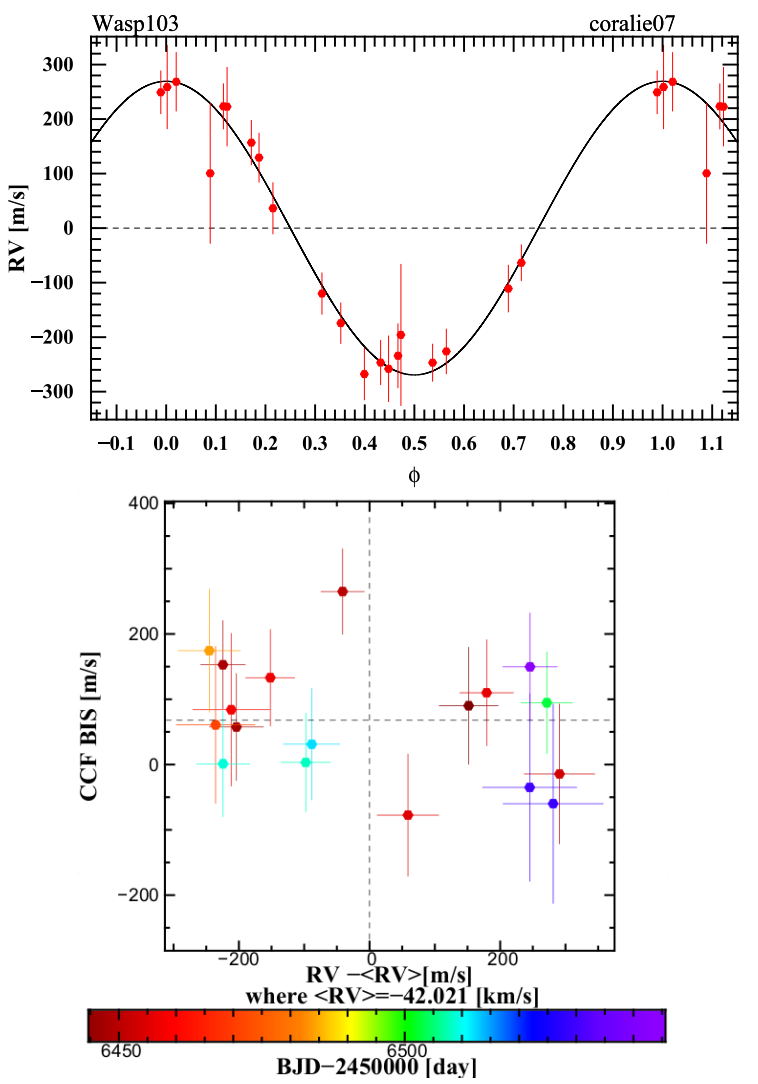

Fig. 3. CORALIE RVs for WASP-103 phase-folded on the best-fit orbital period, and with the best-fit Keplerian model overplotted. Bottom: correlation diagram CCF bisector spans vs. RV. The colors indicate the measurement timings.

( 90 vs. $94 \mathrm{~m} \mathrm{~s}^{-1}$ ), and no evidence for a correlation between the RVs and the bisector spans was found (Fig. 3, lower panel), the slope deduced from linear regression being $-0.06 \pm 0.10$. This bisector analysis allows us to confidently infer that the RV signal is indeed originating from WASP-103.

\section{Analysis}

\subsection{Spectroscopic analysis}

The CORALIE spectra were co-added to produce a single spectrum with an average signa-to-noise ratio $(\mathrm{S} / \mathrm{N})$ of 55 . A spectral analysis was performed using $\mathrm{Fe}$ I and $\mathrm{Fe}$ II equivalent width measurements and the methods described by Doyle et al. (2013). The excitation balance of the Fe I lines was used to determine the effective temperature $\left(T_{\text {eff }}\right)$. The surface gravity $(\log g)$ was determined from the ionization balance of $\mathrm{Fe}$ I and Fe II. The spectroscopic parameters obtained from the analysis are listed in Table 2. The values are quite uncertain because of the low $\mathrm{S} / \mathrm{N}$ of the co-added spectrum.

\subsection{Global analysis}

We performed a global Bayesian analysis of the follow-up photometry and the CORALIE RV measurements, probing the posterior probability distribution functions of the global model parameters with our adaptive Markov chain Monte Carlo (MCMC) algorithm (G13 and references therein).

We performed two MCMC analyses, one assuming a circular orbit and the other an eccentric orbit. A Bayesian model comparison significantly favored the circular model (Bayes factor $>750$
Table 2. Value and $1 \sigma$ errors derived for the WASP-103 star+planet system from our analysis.

\begin{tabular}{|c|c|c|}
\hline Stellar parameters & Value & Unit \\
\hline $\begin{array}{l}\text { RA (J2000) } \\
\end{array}$ & 163715.57 & HH:MM:SS.SS \\
\hline Dec $(\mathrm{J} 2000)$ & +071100.07 & DD:MM:SS.SS \\
\hline$V$-magnitude ${ }^{b}$ & $12.1 \pm 0.01$ & mag \\
\hline$K$-magnitude ${ }^{c}$ & $10.8 \pm 0.02$ & mag \\
\hline Spectral type & F8V & \\
\hline Effective temperature $T_{\text {eff }}$ & $6110 \pm 160$ & $\mathrm{~K}$ \\
\hline Metallicity $[\mathrm{Fe} / \mathrm{H}]$ & $0.06 \pm 0.13$ & dex \\
\hline Lithium abundance $\log A(\mathrm{Li})$ & $2.23 \pm 0.13$ & dex \\
\hline $\operatorname{Age}^{d}$ & $3-5$ & Gyr \\
\hline Projected rotation velocity $V \sin I_{*}$ & $10.6 \pm 0.9$ & $\mathrm{~km} \mathrm{~s}^{-1}$ \\
\hline Microturbulence $V_{r}$ & $1.1 \pm 0.2$ & $\mathrm{~km} \mathrm{~s}^{-1}$ \\
\hline Surface gravity $\log g_{*}$ & $4.22_{-0.05}^{+0.12}$ & $\operatorname{cgs}$ \\
\hline Systemic RV & $-42.001 \pm 0.005$ & $\mathrm{~km} \mathrm{~s}^{-1}$ \\
\hline Density $\rho_{*}$ & $0.414_{-0.039}^{+0.021}$ & $\rho_{\odot}$ \\
\hline Mass $M_{*}$ & $1.220_{-0.036}^{+0.039}$ & $M_{\odot}$ \\
\hline Radius $R_{*}$ & $1.436_{-0.031}^{+0.052}$ & $R_{\odot}$ \\
\hline Luminosity $L_{*}$ & $2.59_{-0.32}^{+0.39}$ & $L_{\odot}$ \\
\hline Distance $d$ & $470 \pm 35$ & parsec \\
\hline Planet parameters & Value & Unit \\
\hline RV semi-amplitude $K$ & $271 \pm 15$ & $\mathrm{~m} \mathrm{~s}^{-1}$ \\
\hline Planet-to-star area ratio $\left(R_{\mathrm{p}} / R_{*}\right)^{2}$ & $1.195_{-0.038}^{+0.042}$ & $\%$ \\
\hline Planet-to-star radius ratio $R_{\mathrm{p}} / R_{*}$ & $0.1093_{-0.0017}^{+0.0019}$ & \\
\hline Transit impact parameter $b$ & $0.19 \pm 0.13$ & $R_{*}$ \\
\hline Transit duration $W$ & $2.593 \pm 0.024$ & hours \\
\hline Time of inferior conjunction $T_{0}$ & $2456459.59957 \pm 0.00075$ & $\mathrm{BJD}_{\mathrm{TDB}}$ \\
\hline Orbital period $P$ & $0.925542 \pm 0.000019$ & $\mathrm{~d}$ \\
\hline Orbital inclination $i_{\mathrm{p}}$ & $86.3 \pm 2.7$ & $\operatorname{deg}$ \\
\hline Orbital semi-major axis $a$ & $0.01985 \pm 0.00021$ & $\mathrm{AU}$ \\
\hline Orbit scale factor $a / R_{*}$ & $2.978_{-0.096}^{+0.050}$ & \\
\hline Mass $M_{\mathrm{p}}$ & $1.490 \pm 0.088$ & $M_{\text {Jup }}$ \\
\hline Radius $R_{\mathrm{p}}$ & $1.528_{-0.047}^{+0.073}$ & $R_{\text {Jup }}$ \\
\hline Surface gravity $\log g_{\mathrm{p}}$ & $3.197_{-0.041}^{+0.036}$ & $\operatorname{cgs}$ \\
\hline Density $\rho_{\mathrm{p}}$ & $0.550_{-0.070}^{+0.061}$ & $\mathrm{~g} \mathrm{~cm}^{-3}$ \\
\hline Equilibrium temperature $^{e} T_{\mathrm{eq}}$ & $2508_{-70}^{+75}$ & K \\
\hline Irradiation & $9.1_{-0.8}^{+1.4} 10^{9}$ & $\operatorname{erg~s}^{-1} \mathrm{~cm}^{-2}$ \\
\hline Roche limit $a_{R}{ }^{f}$ & $0.01695_{-0.00064}^{+0.00085}$ & $\mathrm{AU}$ \\
\hline$a / a_{R}$ & $1.162_{-0.052}^{+0.042}$ & \\
\hline
\end{tabular}

Notes. ${ }^{(a)}$ 2MASS. ${ }^{(b)}$ Egret et al. (1992). ${ }^{(c)}$ Skrutskie et al. (2006). ${ }^{(d)}$ From lithium abundance and stellar evolution modeling. ${ }^{(e)}$ Assuming a null Bond albedo $\left(A_{\mathrm{B}}=0\right)$ and isotropic reradiation $(f=1)$ (Seager et al. 2005). ${ }^{(f)}$ Using $a_{R}=2.46 R_{\mathrm{p}}\left(M_{*} / M_{\mathrm{p}}\right)^{1 / 3}$ (Chandrasekar 1987).

in its favor), so we selected it as our fiducial model. The derived values for the system parameters are shown in Table 2 .

After completing the MCMC analyses, we checked the values derived for the host star WASP-103 by performing a stellar evolution modeling based on the CLES code (Scuflaire et al. 2008). We derived a stellar mass of $1.18 \pm 0.10 M_{\odot}$, in excellent agreement with the MCMC result.

\section{Discussion}

WASP-103 b is a $\sim 1.5$ Jupiter-mass planet orbiting at only $\sim 1.5$ stellar diameter of a late F-type star. Its size is significantly larger than predicted by basic models of irradiated planets. For instance, the models of Fortney et al. (2007) predict a size of $1.25 R_{\text {Jup }}$ for a core-less $1.5 M_{\text {Jup }}$ planet at $0.02 \mathrm{AU}$ from a $1 \mathrm{Gyr}$ solar-twin. The low density of WASP-103 b is probably related 


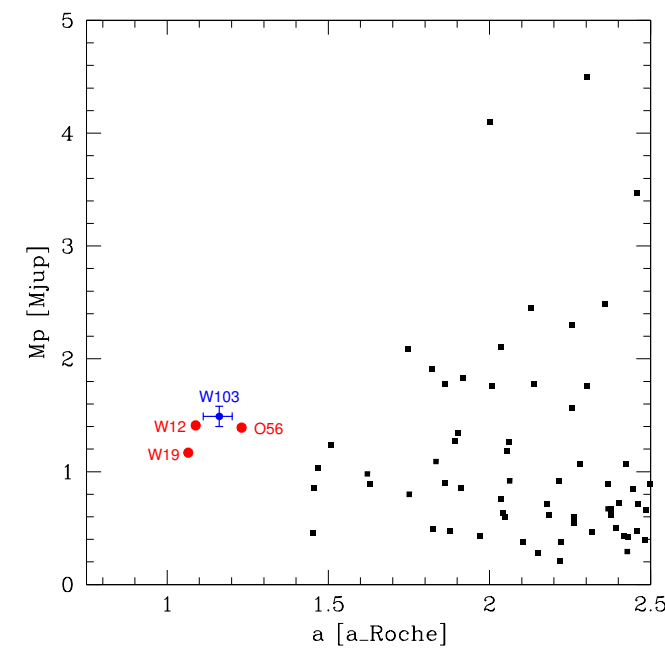

Fig. 4. Diagram planetary mass vs. orbital distance to Roche-limit ratio for known transiting hot Jupiters $\left(M>0.2 M_{\text {Jup }}, P<12 \mathrm{~d}\right.$; data from NASA Exoplanet Archive). O56=OGLE-TR-56b, W12=WASP-12 b, W19=WASP-19 b, and W103=WASP-103 b. Here we only show planets with a ratio of the orbital distance to the Roche-limit lower than 2.5.

not only to its extreme irradiation close to $10^{10} \mathrm{erg} \mathrm{s}^{-1} \mathrm{~cm}^{-2}$, but also to a probably significant level of tidal deformation as its semi-major axis is only 15-20\% larger than its Roche radius. These two features make WASP-103 b join the small subgroup of ultra-short-period gas giants suspected to be just at the edge of tidal disruption, which is composed so far of OGLETR-56 b (Udalski et al. 2002; Konacki et al. 2003), WASP-12 b, and WASP-19b (Fig. 3.2). It has been suggested that these extreme planets might experience Roche-lobe overflow, mass loss (Erkaev et al. 2007; Li et al. 2010; Lai et al. 2010), and significant tidal distortion (Budaj 2011; Leconte et al. 2011). Some signatures of planetary material surrounding WASP-12 have even been detected by Fossatti et al. $(2010,2013)$ and Haswell et al. (2012). It will thus be extremely interesting to search for signs of mass loss and tidal distortion on WASP-103 b, not only to shed new light on the final stages of the life of hot Jupiters. We note, however, that the tidal distortion of the planet probably does not strongly bias the radius measurement presented here. Based on Budaj (2011), we can expect an effective planetary radius ${ }^{2} 2-3 \%$ larger than measured during the transit, which is significantly smaller than our current error bar $(\sim 5 \%)$.

The orbital distribution of hot Jupiters is well explained by assuming an initial formation beyond the ice line followed first by a scattering into an eccentric orbit through the influence of a third perturbing body, then by a slow inward migration and orbital circularization through tidal dissipation (Ford \& Rasio 2006). In this scenario, most scattered planets end up on a circularized orbit at 2-4 Roche radii, and from then their orbits evolve only through the transfer of angular momentum to the star (e.g. Mastumura et al. 2010); the speed of this final evolution depends on the mass of the planet and the tidal dissipation efficiency of the star. In this context, the fact that OGLE-TR-56 b, WASP-12 b, WASP-19 b and WASP-103 b have very similar masses (between 1.1 and $1.5 M_{\text {Jup }}$, see Fig. 3.2) may be meaningful, possibly suggesting a relatively narrow range of tidal dissipation efficiencies for solar-type stars (Chang et al. 2010).

The extreme irradiation of WASP-103 b, its large size and the brightness of its host star make it a member of the select

\footnotetext{
2 I.e. the radius of the sphere with the same volume as the Roche surface of the planet.
}

club of transiting planets amenable for a thorough atmospheric characterization with existing ground-based and space-based facilities (e.g. Seager \& Deming 2010). The comparison of the atmospheric properties of WASP-103 b with those inferred so far for WASP-12 b (e.g. Sing et al. 2013) and WASP-19b (e.g. Anderson et al. 2013) will be particularly useful for assessing the common features and peculiarities among the most extreme hot Jupiters.

Acknowledgements. WASP-South is hosted by the South African Astronomical Observatory and we are grateful for their ongoing support and assistance. Funding for WASP comes from consortium universities and from UK's Science and Technology Facilities Council. TRAPPIST is a project funded by the Belgian Fund for Scientific Research (Fonds de la Recherche Scientifique, F.R.SFNRS) under grant FRFC 2.5.594.09.F, with the participation of the Swiss National Science Fundation (SNF). M. Gillon and E. Jehin are FNRS Research Associates. V. Van Grootel is a FNRS postdoctoral researcher. L. Delrez thanks the Belgian FNRS for funding her Ph.D. thesis. A. H. M. J. Triaud is Swiss National Science Foundation fellow under grant PBGEP2-145594. This research has made use of the NASA Exoplanet Archive, which is operated by the California Institute of Technology, under contract with the National Aeronautics and Space Administration under the Exoplanet Exploration Program.

\section{References}

Anderson, D. R., Smith, A. M. S., Madhusudhan, N., et al. 2013, MNRAS, 430 , 3422

Baranne, A., Queloz, D., Mayor, M., et al. 1996, A\&AS, 119, 373

Budaj, J. 2011, AJ, 141, 59

Charbonneau, D., Brown, T. M., Latham, D. W., \& Mayor, M. 2000, ApJ, 529, L45

Chandrasekhar, S. 1987, Ellipsoidal figures of equilibrium (New York: Dover) Chang, S.-H., Gu, P.-G., \& Bodenheimer, P. H. 2010, ApJ, 708, 1692

Collier-Cameron, A., Pollacco, D., Street, R. A., et al. 2006, MNRAS, 373, 799

Collier-Cameron, A., Wilson, D. M., West, R. G., et al. 2007, MNRAS, 380, 1230

Correia, A. C. M., \& Laskar, J. 2011, in Exoplanets, ed. S. Seager (University of Arizona Press), 239

Doyle, A. P., Smalley, B., Maxted, P. F. L., et al. 2013, MNRAS, 428, 3164

Egret, D., Didelon, P., Mc Lean, B. J., et al. 1992, A\&A, 258, 217

Erkaev, N. V., Kulikov Y. N., Lammer, H., et al. 2007, A\&A, 472, 329

Ford, E. B., \& Rasio, F. A. 2006, ApJ, 638, L45

Fortney, J. J., Marley, M. S., \& Barnes, J. W. 2007, ApJ, 659, 1661

Fossati, L., Haswell, C. A., Froning, C. S., et al. 2010, ApJ, 714, L222

Fossati, L., Ayres, T. R., Haswell, C. A., et al. 2013, ApJ, 766, 20

Gillon, M., Anderson, D. R., Collier-Cameron, A., et al. 2013, A\&A, 552, A82 (G13)

Haswell, C. A., Fossati, L., Ayres, T., et al. 2012, ApJ, 760, 79

Hebb, L., Collier-Cameron, A., Loeillet, B., et al. 2009, ApJ, 693, 1920

Hebb, L., Collier-Cameron, A., Triaud, A., et al. 2010, ApJ, 708, 224

Hellier, C., Anderson, D. R., Collier Cameron, A., et al. 2009, Nature, 460, 1098

Hellier, C., Anderson, D. R., Collier-Cameron, A., et al. 2011a, A\&A, 535, L7

Hellier, C., Anderson, D. R., Collier-Cameron, A., et al. 2011b, in Detection and Dynamics of Transiting Exoplanets, Proceedings of OHP Colloquium, 2327 August 2010, eds. F. Bouchy, R. F. Diaz, \& C. Moutou (Platypus Press), 01004

Hellier, C., Anderson, D. R., Collier-Cameron, A., et al. 2012, MNRAS, 426, 739

Henry, G. W., Marcy, G. W., Butler, R. P., \& Vogt, S. S. 2000, ApJ, 529, L41

Jehin, E., Gillon, M., Queloz, D., et al. 2011, The Messenger, 145, 2

Konacki, M., Torres, G., Jha, S., \& Sasselov, D. D. 2003, Nature, 421, 507

Leconte, J., Lai, D., \& Chabrier, G. 2011, A\&A, 536, A1

Lendl, M., Anderson, D. R., Collier-Cameron, A., et al. 2012, A\&A, 544, A72

Lai, D., Helling, Ch., \& van den Heuvel, E. P. J. 2010, ApJ, 721, 923

Li, S.-L., Miller, N., Lin, D. N. C., \& Fortney, J. J. 2010, Nature, 463, 1054

Matsumura, S., Peale, S. J., \& Rasio, F. A. 2010, ApJ, 725, 1995

Maxted, P. F. L., Anderson, D. R., Collier Cameron, A., et al. 2011, PASP, 123, 547

Pollacco, D. L., Skillen, I., Collier Cameron, A., et al. 2006, PASP, 118, 1407

Queloz, D., Henry, G. W., Sivan, J. P., et al., 2001, A\&A, 379, 279

Scuflaire, R., Theado, S., Montalban, J., et al. 2008, Ap\&SS, 316, 83

Seager, S., \& Deming, D. 2010, ARA\&A, 48, 631

Seager, S., Richardson, L. J., Hansen, B. M. S., et al. 2005, ApJ, 632, 1122

Sing, D., Lecavelier des Etangs, A., Fortney, J., et al. 2013, MNRAS, 436, 2956

Skrutskie, M. F., Cutri, R. M., Stiening, R., et al. 2006, AJ, 131, 1163

Udaksi, A., Zebrun, K., Szymanski, M., et al. 2002, Acta Astron., 52, 115 